\title{
Percepción de la conducta suicida
}

\author{
Perception of suicidal behavior
}

\author{
Gabriel Eduardo Lara - Abada, María Cristina Feregrino-Carreónb, Jesús Baca-Salinasc, \\ Pedro Francisco Méndez-Díazd
}

\begin{abstract}
:
Suicide has become a social issue worldwide by becoming the second leading cause of death among the population between 15 and 29 years according to WHO data. So, an instrument that allows for its timely detection is necessary, the test Psicgarcía (2013) is used and is applied to total population of 20 people and subsequently modified to be standardized, resulting in a reliable test of 51 items
\end{abstract}

Keywords:

Suicidal behavior, suicidal ideation, completed suicide, detection of suicidal behaviour

\section{Resumen:}

El suicidio se ha vuelto una problemática social a nivel mundial al convertirse en la segunda causa de muerte entre la población de entre 15 a 29 años según datos de la OMS. Por lo cual es necesario un instrumento que permite su detección oportuna, se utiliza la prueba de Psicgarcía (2013) la cual se aplica la misma a una población total de 20 personas y posteriormente se modifica para ser estandarizada, dando como resultado una prueba confiable de 51 reactivos.

\section{Palabras Clave:}

Conducta suicida, Ideación suicida, Suicidio consumado, Detección de la conducta suicida.

\section{Introducción}

Objetivo: Evaluar el riesgo de la ideación suicida en la población susceptible mediante la aplicación de un instrumento confiable que permita la detección oportuna de dicha conducta

Método: Procedimiento: Para el pilotaje de la prueba de Psicgarcía (2013) se aplica la misma a una población total de 20 personas de las cuales: son 4 hombres representando el $14.29 \%$ de la población; y 24 mujeres que completan el otro $85.71 \%$. Para los resultados se modifican los ítems en dicotómicos (respuestas de si y no) para que sea posible la captura en el programa de SPSS, pues los resultados eran no válidos y subjetivos. El primer resultado del alfa de Cronbach equivalía a .124 con un total de 62 ítems. Posteriormente se eliminan 6 ítems cuya correlación respecto a las otras era baja, alcanzando así un valor de .290. Al determinar que los reactivos de con quien se vive y la edad no tenían significancia en cuanto al riesgo de cometer el acto suicida, se eliminan, el alfa de Cronbach se eleva entonces hasta .811 .

En el Palacio Legislativo (2019) se planteó la necesidad de implementar y definir políticas públicas que prevengan los suicidios en México. En el marco del Día Mundial para la Prevención del Suicidio, que se conmemora el 10 de septiembre, la diputada Mónica Almeida López planteo la necesidad de garantizar que cada mexicano tenga los recursos mínimos para tener una vida plena y así

a Gabriel Eduardo Lara Abad, Universidad Autónoma del Estado de Hidalgo, Escuela Superior de Atotonilco de Tula, https://orcid.org/00000001-5409-6653, Email: gabriel_lara_9718@uaeh.edu.mx

ь María Cristina Feregrino Carreón, Universidad Autónoma del Estado de Hidalgo, Escuela Superior de Atotonilco de Tula, https://orcid.org/0000-0001-5472-6892, Email: mariacristina_feregrino@uaeh.edu.mx

c Jesús Baca Salinas, Universidad Autónoma del Estado de Hidalgo, Escuela Superior de Atotonilco de Tula, Email: ba377896@uaeh.edu.mx dPedro Francisco Méndez Díaz, Universidad Autónoma del Estado de Hidalgo, Escuela Superior de Atotonilco de Tula, Email: 
combatir algunos detonantes del suicido, como la marginación y la falta de oportunidades. 4

Aseveró que el suicido no es un hecho aislado, sino una serie de acciones que una persona realiza por un tiempo y lo van encaminando a tomar esta decisión. También refirió que en un informe presentado por la Organización Mundial de la Salud (OMS, 2019) se señala, que el ahorcamiento, el auto envenenamiento por pesticidas y el uso de armas de fuego, son los métodos más comunes para cometer suicidio. 1

Los trabajos revisados para este proyecto muestran las estadísticas revisadas sobre las tendencias suicidas en México, y contrastando dichos resultados se obtiene que es entre la población juvenil de 20 a 29 años donde se presenta la mayor cantidad de casos de suicidios consumados (Instituto Nacional de Estadística y Geografía, 2017). Dichas estadísticas son parteaguas de la necesidad de la construcción de un instrumento que permita la detección oportuna del riesgo de dicha población de cometer un acto suicida a cualquier escala y poder ser canalizaos a tiempo.12

Otro dato alarmante se encuentra en un estudio realizado por la OMS en el cual se revela que alrededor de 800000 personas murieron en el año 2012, y el suicidio se determinaba como la segunda causa de muerte entre la población de entre 15 a 29 años. (World Health Organization, 2014).16

El suicidio es un problema grave que no solo afecta a países con altos ingresos, sino que más del $79 \%$ de los suicidios en todo el mundo tuvieron lugar en países de ingresos bajos y medianos, según la OMS cada año, alrededor de 800000 personas se quitan la vida, y muchas más intentan hacerlo. El suicidio no solo afecta a la persona implicada, también tiene repercusiones en sus familias, comunidades e incluso en el país donde este se realiza. Este se puede producir a cualquier edad, y en 2016 fue la segunda causa principal de defunción en el grupo etario de 15 a 29 años en todo el mundo (OMS, 2019).1

En México cada hora y media una persona se quita la vida. De 2010 a 2017 ocurrieron casi 48 mil casos, de los cuales ocho de cada 10 fueron hombres (INEGI, 2017). Poco más de la mitad de las 6 mil 559 personas que se quitaron la vida en 2017 tenían de 15 a 34 años de edad. El suicidio es la segunda causa de muerte de adolescentes de 15 a 19 años de edad, la tercera si se considera hasta los 24 años y la quinta para aquellos que oscilan entre los 10 y 14 años.12

De acuerdo con Narváez, Hernández, Vázquez, Miranda, y Magaña. (2017) en su investigación Depresión e ideas suicidas en jóvenes de educación media superior en Tenosique, Tabasco más de un $50 \%$ de los adolescentes que se suicidan, sufren depresión mayor, un $25 \%$ ha presentado intento de suicidio en algún momento de su vida y solo un $15 \%$ finalmente concluye el acto. Ocho de cada 10 personas que se suicidaron ya lo habían intentado antes y 70 por ciento comentaron sus intenciones con familiares, amigos y médicos. En el caso de los hombres de 20 a 24 años, la tasa de muerte por esta causa por cada 100.000 habitantes es de 9,3.10

En México de acuerdo con un artículo publicado por Infobae (2019) más de la mitad de los suicidios son consumados por personas con trastornos depresivos y cerca de uno de cada cuatro casos de suicidio se asocia al alcoholismo, esquizofrenia 0 ansiedad. Dichos padecimientos requieren un adecuado manejo terapéutico y mayor vigilancia cuando se detecta la ideación suicida. El $40.8 \%$ de los suicidios ocurren en jóvenes de 15 a 29 años.5

En el estado de Hidalgo al año 2018 de acuerdo con el periódico edición nacional se registraron 4 mil 124 suicidios, lo que posiciona a la entidad en el cuarto lugar con mayor incidencia en este problema de salud pública en el país. Siendo el ahorcamiento, estrangulamiento o sofocación, es el método que se utiliza en un 81 por ciento de los casos, seguido por el envenenamiento y disparo de arma con un 8 por ciento. Los usos de objetos cortantes por tendencias suicidas ocurren solo con el uno por ciento de incidencia (2018). Teniendo en cuenta estas estadísticas, lo siguiente será investigar los aspectos que envuelven el suicidio, así como los factores en la población en riesgo.2

\section{El suicidio}

Para comenzar a hablar de suicidio, antes de la parte estadística es necesario definir diversos conceptos del mismo y lo que lo envuelve. El término suicidio proviene de dos expresiones latinas: sui y occidere que significan "matarse a sí mismo". Se lleva a cabo por medio de "conductas suicidas", estas se entienden como toda acción mediante la cual el individuo se causa una lesión, independiente de la letalidad, del método empleado y del conocimiento real de su intención, se produzca o no la muerte del individuo. (Valladolid, 2011) 11

Tenemos que el suicidio, si a primera vista puede parecer un síntoma de patología mental, es mucho más que esto, es un concepto conocido por el individuo normal y posee un valor afectivo y ético, un significado existencial. Pierre Moon genera esta descripción y lo ve como un conjunto de elementos al decir que desde que se inicia la cuestión del suicidio nos encontramos con un problema médico, psicológico y sociológico. Así mismo señala que el suicida nunca está solo en la causa, su acción involucra también el entorno, familia, médico y testigos. El suicidio propiamente dicho significa la muerte del sujeto, las conductas suicidas que responden a la tentativa del suicidio y su finalidad autodestructiva es ambigua por lo general, algunos lo llamaron "para-suicida" (Moon, 1987). 17

Otros conceptos aparecen en las tesis de Bourdin, quien sostiene que el suicidio constituye por sí mismo una entidad morbosa sui-generis. Y la de Esquirol, que considera que es una o varias formas de alienación, que no se manifiesta en personas de mente sana. Ambos 
citados por Durkheim quien creé que, al no existir monomanías, no podrá haber una monomanía suicida y, por consiguiente, el suicidio no es una forma distinta de locura. Un concepto ciertamente vago para los actuales. También propone demostrar que la locura no es una condición necesaria para el suicidio. Los estados psicopáticos que configuran la alienación, no pueden dar razón de la inclinación colectiva al suicidio en sus líneas generales. (Durkheim, 1897).18

El suicidio implica también otros conceptos en sus diferentes formas y es importante comparar lo que cada uno refiere, pues se encuentran en esquemas de acción completamente diferentes. Valladolid (2011) Menciona diversos elementos en cuestión, el suicidio es acto auto infligido para causarse la muerte en forma voluntaria $y / 0$ deliberada, como un proceso que se inicia con la ideación en sus diferentes expresiones (preocupación autodestructiva, planificación de un acto letal, deseo de muerte) después por el intento suicida y finaliza con el suicidio consumado.15

Otro concepto dentro del mismo es el de ideación suicida, que es el resultado de ciertos factores que afectan la vida del sujeto como sentimientos de no poder afrontar una situación abrumadora en su vida, creer que no hay esperanzas en el futuro, en medio de una crisis, pueden sentir que el suicidio es la única salida. Se caracteriza por la presencia de tristeza, pérdida de interés, sentimientos de culpa; dificulta sensiblemente el desempeño en el trabajo o la escuela y la capacidad para afrontar la vida diaria. (Juan Carlos Sánchez Sosa, 2010).3

Barrero (1999) Señala que el espectro completo del comportamiento suicida está conformado por la ideación de autodestrucción en sus diferentes gradaciones: las amenazas, el gesto, el intento y el hecho consumado. El primer punto refiere a la ideación suicida, que abarca un amplio campo de pensamiento que puede adquirir las siguientes formas de presentación: El deseo de morir, la representación suicida, la idea de autodestrucción sin planeamiento de la acción, la idea suicida con un plan indeterminado o inespecífico aún y la idea suicida con una adecuada planificación o plan suicida. El segundo que es el intento suicida, también denominado parasuicidio, tentativa de suicidio, intento de autoeliminación (IAE) o autolesión intencionada (deliberate self harm), se ha definido como aquel acto sin resultado de muerte en el que un individuo, de forma deliberada, se hace daño a sí mismo. Se considera que el intento es más frecuente en los jóvenes, en el sexo femenino, y los métodos más utilizados son los suaves o no violentos, principalmente la ingestión de fármacos o sustancias tóxicas.11

El suicidio consumado abarca todos aquellos actos lesivos autoinfligidos con resultado de muerte. Son más frecuentes en adultos y ancianos, en el sexo masculino, y los métodos preferidos son los llamados duros 0 violentos, como las armas de fuego, el ahorcamiento, el fuego, etcétera (Barrero S. A., 1999).11

\section{Marco legal del suicidio}

El suicidio se ha convertido en un problema de salud pública que afecta a las personas, sus familias y a la comunidad por lo cual es necesario legislar sobre este problema con el propósito de prevenirlo y contenerlo. Al presentar la iniciativa de ley en la Gaceta Parlamentaria (2012) Mondragón menciona que el suicidio es la única muerte que puede ser prevenida, aunque no se pueden prevenir todos los suicidios, pero sí la mayoría. 7

La Organización Mundial de la Salud cuenta con evidencia de que el suicidio se puede evitar tomando en cuenta estrategias que contemplan la restricción de acceso a métodos comunes de suicidio, por ejemplo, armas de fuego y sustancias tóxicas como plaguicidas, han demostrado ser eficaces para reducir las tasas de suicidio. No obstante, se deben adoptar enfoques multisectoriales con muchos niveles de intervención y actividades, además, de que datos disponibles demuestran que la prevención y el tratamiento adecuados de la depresión y del abuso de alcohol y de sustancias reducen las tasas de suicidio, al igual que el contacto de seguimiento con quienes han intentado suicidarse (2012).13

Según Borges, Orozco, Benjet, y Medina (2010) en su investigación "Suicidio y conductas suicidas en México: retrospectiva y situación actual", el estudio de las conductas suicidas, desde el punto de vista epidemiológico, es diferente al suicidio consumado, que se basa en gran medida en estadísticas recolectadas rutinariamente por las instancias oficiales a través del certificado de defunción. En el caso del intento de suicidio, no hay organismo que disponga de información veraz sobre este problema ya que no es obligatorio reportar/o, mucho menos el reportar la ideación o los planes suicidas. Por ello resulta importante establecer el deber normativo de llevar un registro de este aspecto de la conducta suicida a fin de contar con una base sólida para construir la política de prevención, tal y como se propone en la iniciativa de ley que adiciona los artículos 73 Bis a 73 Bis 2 a la Ley General de Salud. 6

La prevención del suicidio se establece en el ámbito de la salud mental. De acuerdo con el artículo 20. y el 3o., fracción VI, de la Ley General de Salud (2006) adscrita a la Constitución Política de los Estados Unidos Mexicanos, entre las finalidades del derecho a la protección de la salud se encuentran el bienestar físico y mental del hombre, para contribuir al ejercicio pleno de sus capacidades y la prolongación y mejoramiento de la calidad de la vida humana. Asimismo, se reconoce a la salud mental como materia de salubridad general, por lo que la intervención activa y eficaz del Estado debe llevarse a cabo de manera obligatoria.8

Es importante que en las políticas públicas se incluyan a las escuelas, centros de trabajo y organizaciones sociales y vecinales para que se fortalezca la expectativa de resultados, como podemos observar la prevención del suicidio requiere también la intervención de sectores distintos como los ya mencionados con anterioridad y exige un enfoque innovador, integral y multisectorial, con la participación de ciertos sectores, como por ejemplo los 
de la educación, el mundo laboral, la policía, la justicia, la religión, el derecho, la política y los medios de comunicación (Organización Mundial de la Salud, 2012).13

La iniciativa de ley que adiciona los artículos 73 Bis a 73 Bis 2 a la Ley General de Salud (2011) tiene como objetivo que el Estado, a través de las autoridades competentes, impulse la organización de redes sociales para la prevención del suicidio, al igual que la reforma constitucional en materia de derechos humanos, publicada por parte de la Secretaria de Gobernación, establece la obligación de las autoridades de los tres órdenes de gobierno de respetar, proteger, fomentar y garantizar los derechos humanos en la república, para que se puedan cumplir con las nuevas obligaciones, aplicadas a los servidores públicos del sector de la salubridad general, como el requerimiento de mayor atención y derechos específicos a las personas en riesgo de suicidio y sus familias. 9

Así el decreto emitido en la Gaceta Parlamentaria (2012) adiciona el artículo 73 Bis a la Ley General de Salud, además de los artículos 73 Bis, 73 Bis 1 y 73 Bis 2 para quedar como sigue:

- Artículo 73 Bis. Corresponde a la Secretaria de Salud en coordinación con los Gobiernos de las Entidades Federativas en materia de prevención de trastornos mentales y del comportamiento que desembocan en actos tentativos 0 consumados de suicidio:

- Promover y apoyar la investigación de las causas del suicidio y de la conducta suicida;

- II. Realizar acciones programáticas y de prevención del suicidio, dirigidas a toda la población y en particular a los grupos considerados de alto riesgo.

- Artículo 73 Bis 1. Corresponde a la Secretaría de Salud emitir las disposiciones de carácter general para la detección control y vigilancia epidemiológica de los trastornos mentales y del comportamiento que pueden derivar en intento de suicidio o suicidio consumado, entre los usuarios de los servicios de atención médica, así como, organizar y conservar la información que se genere, con base en indicadores epidemiológicos, para efectos de la construcción de instrumentos de política pública en la materia.

- Artículo 73 Bis 2. La Secretaría de Salud establecerá los criterios para la atención médica y de urgencia de las personas con tendencias suicidas, así como, para el acompañamiento y la orientación oportuna de sus familiares, conformidad con las Normas Oficiales Mexicanas que al efecto emita.7

\section{Aspectos generales del suicidio}

Entre los casos propuestos en un estudio de Tanatología (Ambriz, Hernández, y Reyes, 2011) a examinar para la detección temprana de adolescentes con riesgo suicida, propone los siguientes aspectos:

1. Comportamiento suicida. Debe investigarse los deseos de morir, la idea suicida, los gestos, las amenazas y el plan de autodestrucción, el método que va a emplear, circunstancias en que lo realizaría, etcétera.

2. Ámbito familiar. Relaciones conflictivas con padres, violencia familiar, padres con enfermedades mentales, familiares con conducta suicida y presencia de amistades con este comportamiento.

3. Cuadro clínico. Explorar signos de enfermedad mental, especialmente depresión, esquizofrenia y conducta suicida previa del adolescente.

4. Estado psicológico. Precisar la presencia de desesperanza, sentimientos de soledad, angustia, culpabilidad, abuso de alcohol, depresión, rabia, agresividad.

5. Motivos. Pérdida de una relación valiosa, conflictos escolares, humillaciones, problemas familiares, deseos de matarse, etcétera.14

En otra publicación se describen otras formas usuales de la conducta suicida, y son el intento de suicidio y el acto de suicidio como tal. Sin embargo, es necesario considerar otros aspectos aparentemente no tan relevantes pero que pudiesen ser indicadores importantes para su detección, pues los posibles pensamientos son variados y pueden ser:

El deseo de morir, la representación suicida (imaginación), la idea de autodestrucción sin planeamiento de la acción, la idea suicida con un plan indeterminado o inespecífico aún y la idea suicida con una adecuada planificación (Barrero, 1999).11

\section{Instrumento para la detección de la conducta suicida}

Tomando como referente el cuestionario Psicgarcia (2013) se desarrolló una base de datos con ayuda del programa SPSS V 25, posteriormente los datos correspondientes de los 28 participantes se capturaron en el programa y se inició el análisis de los datos.

Se usó la Rho de Pearson para correlacionar los ítems e identificar los ítems con correlaciones significativas entre ellos, así de un total de 62 ítems, se excluyeron 6 ítems que tenían correlaciones muy pobres, y se eliminaron dos elementos de la información general que no tenían correlación en cuanto a cometer el acto suicida.

De acuerdo a estas correlaciones agrupamos ítems afines entre ellos de acuerdo al nivel de significancia bilateral que obtuvieron para identificar qué es lo que se desea obtener con ellos, así se propuso un total de 3 índices, cada uno con sus respectivos indicadores y estos a su vez con la tentativa del total de ítems en cada uno de ellos, tal como se muestra en la tabla 1.1. 
La prueba final se compone de 51 reactivos mediante los siguientes indicadores:

\begin{tabular}{|l|l|c|}
\hline Índicadores & Variables & No. de ítems \\
\hline \multirow{3}{*}{ 1. Antecedentes } & 1.1 Vivencias & 5 \\
\cline { 2 - 3 } & 1.2. Conductas & 5 \\
\cline { 2 - 3 } & 1.3. Adicciones & 4 \\
\hline \multirow{2}{*}{ 2. Contexto } & 2.1. Convivencia & 5 \\
\cline { 2 - 3 } & 2.2. Eventos & 3 \\
\hline \multirow{2}{*}{$\begin{array}{l}\text { 3. Lo que hay } \\
\text { detrás del }\end{array}$} & 3.1. Emociones & 13 \\
\cline { 2 - 3 } suicidio & 3.2. Pensamiento & 6 \\
\cline { 2 - 3 } & 3.3. Perspectivas & 10 \\
\hline
\end{tabular}

Tabla 1: Índice del instrumento sobre percepción suicida

(Baca y Méndez, 2019)

\section{Referencias}

[1] Organización Mundial de la Salud. (2019). Suicidio. Recuperado de www.who.int/es/news-room/factsheets/detail/suicide

[2] (21 de febrero de 2018). Qué miedo: Hidalgo es cuarto lugar en suicidios. Edición nacional. Recuperado de https://www.am.com.mx/hidalgo/noticias/Que-miedo-Hidalgoes-cuarto-lugar-en-suicidios-20180220-0001.html

[3] Sánchez, J.C., Musita, G., Villareal, M.E y Martínez, B. (2010). Ideación Suicida en Adolescentes: Un Análisis Psicosocial. Intervención psicosocial, 3(19), 279-287. Recuperado

de

http://scielo.isciii.es/pdf/inter/v19n3/v19n3a08.pdf

[4] Palacio legislativo. (2019). Nota $N^{\circ}$. 3040. Recuperado de http://www5.diputados.gob.mx/index.php/camara/Comunicacio n/Agencia-de-Noticias/2019/Septiembre/10/3040-Plantea-

Almeida-Lopez-la-necesidad-de-implementar-y-definir-

politicas-publicas-que-prevengan-los-suicidios-en-Mexico

[5] (10 de septiembre de 2019). Suicidio: la segunda causa de muerte en México en jóvenes de 15 a 19 años de edad, pero sólo un hospital especializado pediátrico en el país. Infobae. Recuperado

https://www infobae.com/america/mexico/2019/09/10/suicidiola-segunda-causa-de-muerte-en-mexico-en-jovenes-de-15-a-19anos-de-edad-pero-solo-un-hospital-especializado-pediatricoen-el-pais/

[6] Borges, G., Orozco, R., Benjet, C \& Medina, M.E. (2010). Suicidio y conductas suicidas en México: Retrospectiva y situación actual. Salud pública de México, 52(4), 292-304. Recuperado

http://www.scielo.org.mx/pdf/spm/v52n4/v52n4a05.pdf

[7] Iniciativa que adiciona los artículos 73 Bis a 73 Bis 2 a la Ley General de Salud. (2012, 15 de noviembre). Gaceta Parlamentaria, Número 3646-III. Noviembre 15, 2010. Recuperado

http://gaceta.diputados.gob.mx/Black/Gaceta/Anteriores/62/20 12/nov/20121115-III/Iniciativa-18.html

[8] Ley general de salud. (2006, 26 de abril). Constitución Política de los Estados Unidos Mexicanos. Abril 26, 2006.
Recuperado

\section{de}

http://www.salud.gob.mx/unidades/cdi/legis/lgs/LEY_GENER AL_DE_SALUD.pdf

[9] Decreto por el que se modifica la denominación del Capítulo I del Título Primero y reforma diversos artículos de la Constitución Política de los Estados Unidos Mexicanos. (2011, 10 de junio). Constitución Política de los Estados Unidos Mexicanos. Junio 10, 2011. Recuperado de https://www.dof.gob.mx/nota_detalle.php?codigo $=5194486 \& f e$ cha $=10 / 06 / 2011$

[10] Narváez, Y., Hernández, M.I., Vázquez, M., Miranda, A. \& Magaña, L. (2017). Depresión e ideas suicidas en jóvenes de educación media superior en Tenosique, Tabasco. Salud en Tabasco, 2(23), 28-33. Recuperado de http://www.redalyc.org/pdf/487/48754566005.pdf

[11] Barrero, D. S. (1999). El suicidio, comportamiento y prevención. La Habana, Cuba: Revista Cubana de Medicina General Integral.

[12] Instituto Nacional de Estadística y Geografía.

(2017). "Estadísticas a Propósito del Día Mundial para la Prevención del Suicidio". México.

[13] Organización Mundial de la Salud. (agosto de 2012). who.int. Obtenido de https://www.who.int/mental_health/prevention/suicide/suicidep revent/es/

[14] Ambriz, R.G., Hernández, Y.G. y Reyes, J.P. (2011). Suicidio en adolescentes. Asociación Mexicana de Tanatología, A.C.

[15] Valladolid, M. N. (2011). Suicidio. Revista Peruana de Epidemiología, pp. 88-81.

[16] World Health Organization. (2014). Preventing Suicide: A Global Imperative. Geneva. [17] Moon, P. (1987). El suicidio. Publicaciones Cruz O, SA.

[18] Durkheim, E. (1989). El suicidio (Vol. 37). Ediciones Akal. 